\title{
Review: non-invasive interventions improve symptoms and psychological functioning in patients with lung cancer
}

Solà I, Thompson E, Subirana M, et al. Non-invasive interventions for improving well-being and quality of life in patients with lung cancer. Cochrane Database Syst Rev 2004;(4):CD004282.

\section{A Are non-invasive interventions delivered by healthcare professionals effective for improving symptoms, psychological functioning, and quality of life in patients with lung cancer?}

\section{METHODS}

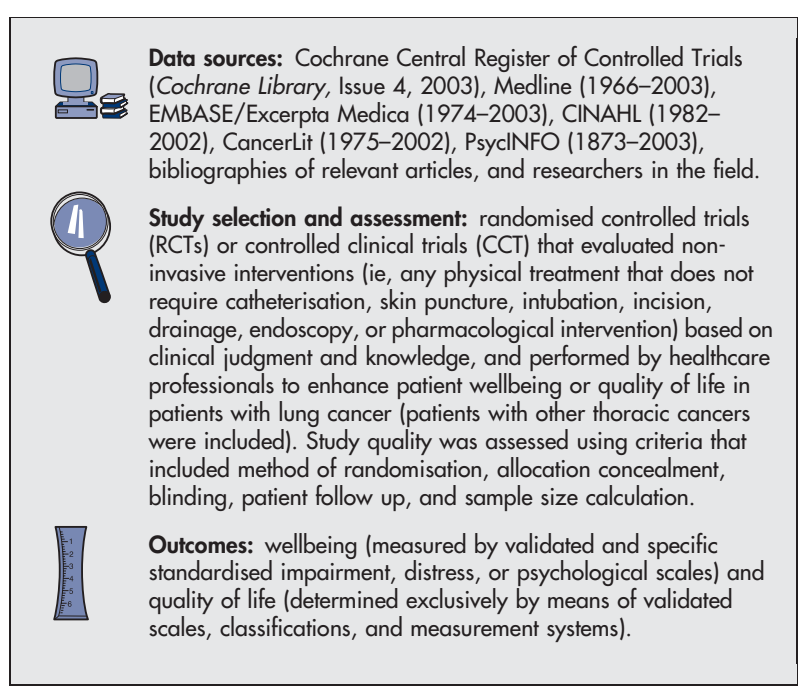

\section{MAIN RESULTS}

9 RCTs met the selection criteria and were categorised into 6 groups. Meta-analysis was not done because of study heterogeneity. (1) Nurse led management of breathlessness programme $v$ usual care (2 RCTs, $n=143$ ). Improvement in symptoms, performance status, and emotional functioning was greater in the intervention groups than in the control groups ( $\mathrm{p}$ values <0.05). (2) Nurse led follow up programmes $v$ standard physician follow up ( 3 RCTs, $n=370$ ). Structured nursing follow up programmes were associated with positive effects on delay in clinical deterioration, dependency, symptom distress, emotional functioning, and satisfaction with care ( $\mathrm{p}$ values $<0.05$ ). (3) Nutritional advice by a dietician to achieve targeted caloric intake $v$ ad lib oral diet without support from a dietician (1 RCT, $n=192$ ). Nutritional advice increased caloric intake $(\mathrm{p}<0.001)$ but not weight or survival. (4) Counselling $v$ no intervention ( $1 R C T, n=65)$. Counselling was associated with improvements in depression, alienation score, life satisfaction, and self esteem throughout follow up ( $p$ values $<0.05$ ). However, the groups did not differ for functional status, degree of impairment, or survival. (5) Preoperative exercise (walking and stair climbing; and arm, leg, and breathing exercises) $v$ no intervention (1 RCT,

For correspondence: Mr I Solà, lberoamerican Cochrane Centre, Hospital de la Santa Creu i Sant Pau, Barcelona, Spain. isola@santpau.es

Source of funding: Ministerio de Sanidad y Consumo, Instituto de Salud Carlos III, Spain. $n=104$ ). Increase in "power" (capacity of an individual to knowingly participate in change) was greater in the intervention group than in the control group $(\mathrm{p}<0.001)$. (6) 30 minutes of foot reflexology $v$ no intervention ( $1 R C T, n=10)$. Reduction in anxiety scores was greater in the intervention group than in the control group $(\mathrm{p}=0.002)$.

\section{CONCLUSION}

Non-invasive interventions of various types delivered by healthcare professionals are effective for improving symptoms and psychological functioning in patients with lung cancer.

\section{Commentary}

7 he World Health Organization predicts a steep international increase in the number of new patients with lung cancer from 10 million in 2000 to 15 million in 2020. ${ }^{1}$ With such a worrisome prospect in mind, the systematic review by Sola et al is timely. Study strengths include a comprehensive search strategy, examination of outcomes that are linked to supportive care, and quality assessment scoring.

A narrative synthesis was done because of intervention and outcome heterogeneity, but the development of 6 intervention categories for the 9 studies was somewhat artificial. In addition, studies that included only patients with lung cancer had relatively small samples (34-202 patients) and insufficient follow up in some cases. However, promising interventions included breathlessness management, ${ }^{23}$ exercise, ${ }^{4}$ and nurse led programmes (excluding structured assessment).

The most valuable contribution of the review by Sola et al is the focus and attention drawn to this broad, methodologically difficult, and often neglected area of study. Although most programmatic research emphasises prevention and medical management, supportive care interventions focusing on management of the effects of lung cancer or treatment on individuals, their families, and the healthcare system must be considered a research imperative. Practitioners and health planners need well conceived and rigorous research in order to provide comprehensive and up to date biopsychosocial care in home, community and institutional settings.

E Ann Mohide, RN, MHSc, MSc McMaster University, School of Nursing Hamilton, Ontario, Canada

1 World Health Organization. Global cancer rates could increase by $50 \%$ to 15 million by 2020. Accessed 16 January 2005. http://www. who.int/ mediacentre/news/releases/2003/pr27/en/

2 Corner J, Plant H, A'Hern R, et al. Non-pharmacological intervention for breathlessness in lung cancer. Palliat Med 1996;10:299-305.

3 Bredin M, Corner J, Krishnasamy M, et al. Multicentre randomised controlled trial of nursing intervention for breathlessness in patients with lung cancer. BMJ 1999;318:901-4.

4 Wall $L M$. Changes in hope and power in lung cancer patients who exercise. Nurs Sci Q 2000;13:234-42.

5 Sarna L. Effectiveness of structured nursing assessment of symptom distress in advanced lung cancer. Oncol Nurs Forum 1998;25:1041-8. 